\title{
Addendum
}

\section{Chain equivalences for symplectic bases, quadratic forms and tensor products of quaternion algebras}

\author{
[J. Algebra Appl., Vol. 14, No. 3 (2015) 1550030] \\ Adam Chapman \\ Department of Mathematics \\ Michigan State University \\ East Lansing, MI 48824 \\ adam1chapman@yahoo.com \\ Received 13 January 2015 \\ Accepted 13 January 2015 \\ Published 23 April 2015
}

Communicated by L. H. Rowen

\begin{abstract}
We show how the chain lemma for quaternion algebras over fields of char $F \neq 2$ and $I_{q}^{3} F=0$ can be strengthened so that up to two slots are changed at a time.
\end{abstract}

Theorem 0.1 [1, Theorem 5.2]. If char $F \neq 2$ and $I_{q}^{3} F=0$ then every two isomorphic tensor products of $n$ quaternion algebras are connected by a finite number of steps, where each step is either

(1) $\left(c_{k}, d_{k}\right) \otimes\left(c_{k+1}, d_{k+1}\right) \rightarrow\left(c_{k},\left(\beta^{2}-c_{k} c_{k+1}\right) d_{k}\right) \otimes\left(c_{k+1},\left(\beta^{2}-c_{k} c_{k+1}\right) d_{k+1}\right)$

for some $k \in\{1, \ldots, n-1\}$ and $\beta \in F$, or one of the following types:

(2) $\left(c_{k}, d_{k}\right) \rightarrow\left(\left(\alpha^{2}-\beta^{2} d_{k}\right) c_{k}, d_{k}\right)$,

(3) $\left(c_{k}, d_{k}\right) \rightarrow\left(c_{k},\left(\alpha^{2}-\beta^{2} c_{k}\right) d_{k}\right)$,

for some $k \in\{1, \ldots, n\}$ and $\alpha, \beta \in F$.

Proof. The proof is essentially the proof of [1, Theorem 5.2], with the following observation: There is one additional type of step in the original theorem

$$
\left(c_{1}, d_{1}\right) \otimes \ldots \otimes\left(c_{n}, d_{n}\right) \rightarrow\left(c_{1},\left(\beta^{2}-\prod_{r=1}^{n} c_{r}\right) d_{1}\right) \otimes \ldots \otimes\left(c_{n},\left(\beta^{2}-\prod_{r=1}^{n} c_{r}\right) d_{n}\right) .
$$

This step corresponds to a change of the last two entries $\left\langle a_{n+1}, b_{n+1}\right\rangle$ in the corresponding quadratic form. Now, we can avoid such changes, because both the initial 


\section{A. Chapman}

and final quadratic forms can be chosen so that they have -1 in the last entry, and therefore according to the Witt cancellation theorem they are connected by a chain of steps that leave the last entry unchanged.

\section{Acknowledgements}

The author started considering improving this theorem as a result of a question Vladimir Chernousov raised in a lecture at the second joint international meeting of the IMU and the AMS.

\section{Reference}

[1] A. Chapman, hain equivalences for symplectic bases, quadratic forms and tensor products of quaternion algebras, J. Algebra Appl. 14(3) (2015) 1550030 (9 pages). 